Camila da Silva Conceição

Análise crítica dos rótulos de alimentos orgânicos embalados

Dissertação de Mestrado

Dissertação apresentada como requisito parcial para obtenção do título de Mestre pelo Programa de PósGraduação em Metrologia (Área de concentração: Metrologia para Qualidade e Inovação) da PUC-Rio.

Orientadora: Prof. ${ }^{a}$ Maria Fatima Ludovico de Almeida 

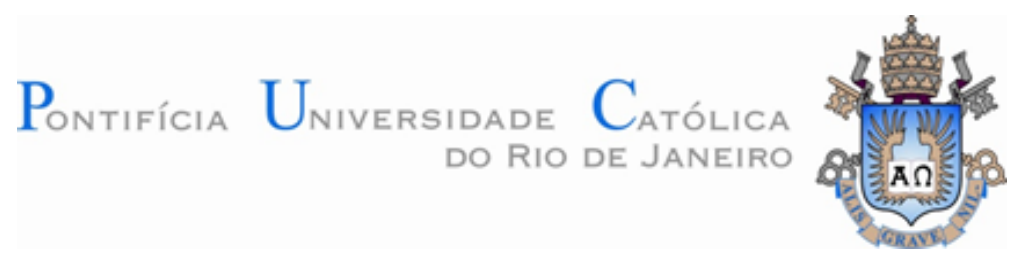

Camila da Silva Conceição

\section{Análise crítica dos rótulos de alimentos orgânicos embalados}

Dissertação apresentada como requisito parcial para obtenção do título de Mestre pelo Programa de Pós-Graduação em Metrologia da PUC-Rio. Aprovada pela Comissão Examinadora abaixo assinadas.

Prof.- Maria Fatima Ludovico de Almeida

Presidente/ Orientadora

Programa de Pós-Graduação em Metrologia (PUC-Rio)

Prof. Maurício Nogueira Frota

Programa de Pós-Graduação em Metrologia (PUC-Rio)

Prof. ${ }^{\text {a }}$ Maria Fernanda de A. Costa Fonseca

Empresa de Pesquisa Agropecuária do Estado do Rio de Janeiro

Prof. José Eugenio Leal

Coordenador Setorial de Pós-Graduação do

Centro Técnico Científico (PUC-Rio)

Rio de Janeiro, 27 de dezembro de 2012 
Todos os direitos reservados. É proibida a reprodução total ou parcial do trabalho sem autorização da universidade, da autora e de seus orientadores.

\section{Camila da Silva Conceição}

Graduada em Economia Doméstica pela Universidade Federal Rural do Rio de Janeiro (2007). Mestranda do Programa de PósGraduação em Metrologia para Qualidade e Inovação.

Ficha Catalográfica

Conceição, Camila da Silva

Análise crítica dos rótulos de alimentos orgânicos embalados / Camila da Silva Conceição; orientadora: Maria Fatima Ludovico de Almeida.- Rio de Janeiro: PUC, Programa de Pós-Graduação em Metrologia, 2012.

v.1, 130 f. : il. (color.) ; $30 \mathrm{~cm}$

1. Dissertação (mestrado)-Pontifícia Universidade Católica do Rio de Janeiro, Programa de Pós-Graduação em Metrologia, Centro Técnico Científico, 2012.

Inclui referências bibliográficas e anexos

1. Metrologia - Teses. 2. Rotulagem de alimentos embalados. 3. Avaliação da conformidade. 4. Regulamentação de alimentos embalados. 5. Alimentos orgânicos embalados. 6. Certificação de alimentos orgânicos. I. Almeida, Maria Fatima Ludovico de. II. Pontifícia Universidade Católica do Rio de Janeiro. Programa de Pós-Graduação em Metrologia. Área de concentração: Metrologia para Qualidade e Inovação. III. Título.

CDD: 389.1 


\section{Agradecimentos}

A Deus, que me dá forças para seguir em busca dos meus sonhos.

Aos meus pais e irmão, por sempre me incentivarem e apoiarem em todas as minhas decisões.

À minha orientadora Professora Fatima Ludovico, pela confiança e preciosa orientação, que muito me ajudou na realização deste trabalho.

A todos os professores do Programa de Pós-graduação em Metrologia para Qualidade e Inovação (PósMQI) e, em especial, ao Professor Maurício Frota, pela oportunidade de desenvolvimento profissional e acadêmico.

Aos membros da banca pela disponibilidade e sugestões construtivas que muito irão enriquecer esse trabalho.

Aos colegas de classe pela amizade, por todo auxílio, compreensão e por me apoiarem na caminhada deste curso.

Aos amigos que conquistei Alex, Ana Carolina, Antônio Fernando, Bruno, Juan, Khrissy e Laura muito obrigada pelo apoio e incentivo constante.

As minhas amigas Cintia Carraro e Francine Rossone, que foram as primeiras a me incentivarem nessa iniciativa.

A minha amiga Juliana Nóbrega que me acolheu no Rio de Janeiro no início desta jornada.

Ao meu amigo e companheiro Augusto Almeida, por me ajudar, apoiar e incentivar sempre, com muito amor e carinho.

A todos os amigos pelos momentos de descontração, afeto e compreensão.

Às funcionárias da Secretaria do PósMQI, Márcia e Paula, por serem sempre prestativas quando precisei.

À CAPES e à PUC-Rio, pelo apoio financeiro, sem o qual não seria possível esta realização pessoal.

E a todos que, de alguma forma, contribuíram para a realização deste trabalho. 


\section{Resumo}

Conceição, Camila da Silva; Almeida, Maria Fatima Ludovico de (Orientadora). Análise crítica dos rótulos de alimentos orgânicos embalados. Rio de Janeiro, 2012. 130 p. Dissertação de Mestrado Programa de Pós-Graduação em Metrologia. Área de concentração: Metrologia para Qualidade e Inovação, Pontifícia Universidade Católica do Rio de Janeiro.

O Brasil encontra-se entre os maiores produtores de orgânicos do mundo e o desenvolvimento do mercado de alimentos orgânicos está diretamente relacionado à confiança dos consumidores quanto à origem dos produtos. Nesse contexto, as informações dos rótulos e o selo de garantia de produto orgânico facilitam o consumidor identificar os alimentos que estão em conformidade com os regulamentos e normas técnicas vigentes e criam ambiente de confiança em circuitos longos de comercialização para os mercados desses produtos. Considerando a relevância do tema e a escassez de pesquisas de campo sobre rotulagem de alimentos orgânicos, o objetivo da dissertação é analisar a situação da conformidade de rótulos de alimentos orgânicos embalados e comercializados no município do Rio de Janeiro em relação à legislação aplicável, e propor recomendações para as entidades interessadas em uma melhor adequação da rotulagem desses produtos. A pesquisa de campo foi conduzida em 2012 e as amostras analisadas totalizaram 107 produtos, classificados segundo sete categorias, a saber: conservas e antepastos; confeitaria e chocolataria; bebidas; chás; laticínios; barras; e biscoitos e snacks. Os resultados quantitativos e qualitativos da conformidade (e da não-conformidade) da rotulagem das categorias selecionadas poderão ser utilizados por diversas entidades de interesse, como órgãos reguladores, órgãos de defesa do consumidor, organizações nãogovernamentais, fabricantes e seus fornecedores e consumidores de alimentos orgânicos embalados.

\section{Palavras-chave}

Metrologia; rotulagem de alimentos embalados; avaliação da conformidade; regulamentação de alimentos embalados; alimentos orgânicos embalados; certificação de alimentos orgânicos. 


\section{Abstract}

Conceição, Camila da Silva; Almeida, Maria Fatima Ludovico de (Advisor).

Critical analysis of packaged organic food labels. Rio de Janeiro, 2012. 130p. MSc. Dissertation - Programa de Pós-Graduação em Metrologia. Área de concentração: Metrologia para Qualidade e Inovação, Pontifícia Universidade Católica do Rio de Janeiro.

Brazil is among the largest producers of organic food and the development of organic food market is directly concerned to consumer confidence in relation to authenticity of the products. In this context, food labels and organic certification seals provide a wide range of valuable information to make healthier choices easier to consumers and also reliability in long circuits of organic food to market. Considering the importance of the topic and the lack of research with this focus, The objective of this dissertation is two-fold: (i) to analyze the state of compliance of packaged organic food labels commercialized in the city of Rio de Janeiro, focusing on the mandatory labeling information; and (ii) to propose recommendations for organizations interested in compliance of packaged organic food labeling to current Brazilian regulation concerning the selected food categories. The field research was conducted in 2012 and the samples analyzed totaled 107 products, classified into seven categories, as follows: canned and antepastos; confectionery and chocolates; beverages; teas; dairy products; biscuits and snacks; and cereal bars. The quantitative and qualitative results of compliance (and noncompliance) labeling of the selected categories can be used by several entities of interest such as regulators, consumer protection agencies, nongovernmental organizations, manufacturers and their suppliers and organic food consumers.

\section{Keywords}

Metrology; labeling of packaged food; conformity assessment; packaged food regulation; packaged organic food; organic food certification. 


\section{Sumário}

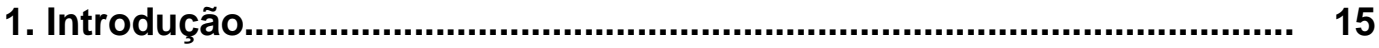

1.1 Definição do problema de pesquisa................................................... 18

1.2 Objetivos: geral e específicos........................................................... 19

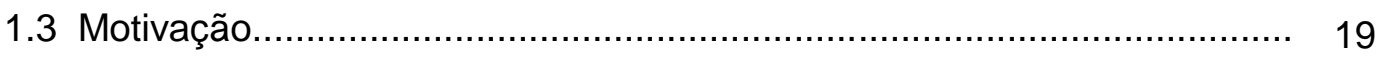

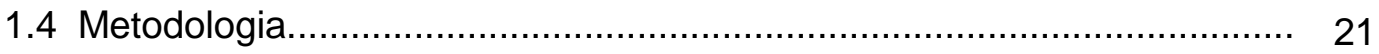

1.4.1 Fase exploratória e descrtitiva...................................................... 22

1.4.2 Pesquisa aplicada................................................................... 23

1.4.3 Fase conclusivo-propostiiva........................................................ 25

1.5 Estrutura da dissertação............................................................... 25

2. Qualidade e segurança de alimentos: um olhar para os alimentos orgânicos.

2.1 Segurança alimentar versus segurança dos alimentos: conceitos básicos.

2.2 Atuação do Codex Alimentarius.......................................................... 31

2.3 Breve histórico dos sistemas de gestão da qualidade e segurança de alimentos............................................................................................ 32

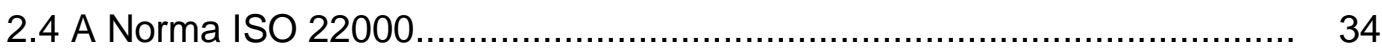

2.4.1 Programa de Pré-Requisitos (PPR) .............................................. 35

2.4.2 O Plano de Análise de Perigos e Pontos Críticos de Controle (APCC).. 36

2.5 Importância da rotulagem de alimentos embalados................................. 37

2.6 Alimentos orgânicos embalados: regulamentação, rotulagem e

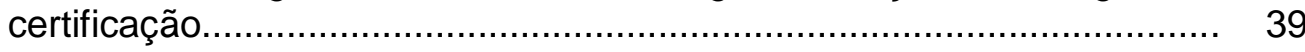

2.6.1 Regulamentação dos alimentos orgânicos...................................... 41

2.6.2 Organismos de avaliação.............................................................. 45

2.6.3 Certificados de conformidade orgânica............................................. 45

2.6.4 Rotulagem de alimentos orgânicos par ao mercado interno................. 49

2.7 Considerações finais sobre o capítulo.................................................. 51

3. Análise comparativa das regulamentações de alimentos embalados: foco nas informações obrigatórias................................................... 53

3.1 Comunidade Européia................................................................ 53

3.2 Estados Unidos.............................................................................. 57 
3.3 Canadá

3.4 Austrália e Nova Zelândia.................................................................. 60

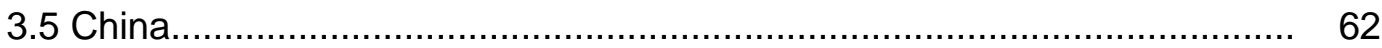

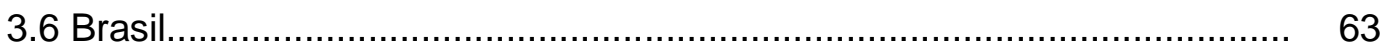

3.7 Considerações finais sobre o capítulo..................................................... 65

4. Avaliação de rótulos de alimentos orgânicos de categorias

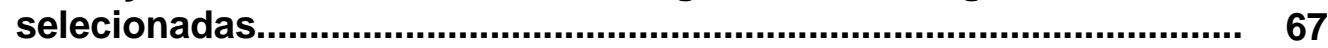

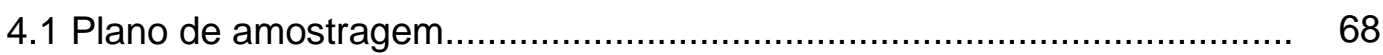

4.2 Regulamentação utilizada para a verificação da conformidade dos

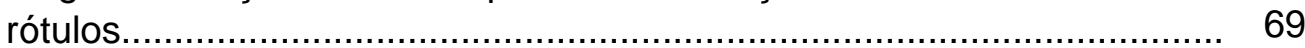

4.3 Itens de rotulagem e parâmetros para verificação da conformidade dos

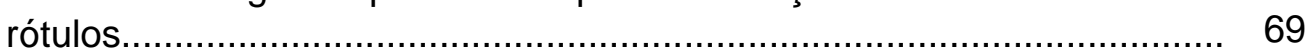

4.4 Coleta, tratamento e análise dos dados ................................................ 72

4.5 Discussão dos resultados.................................................................. 73

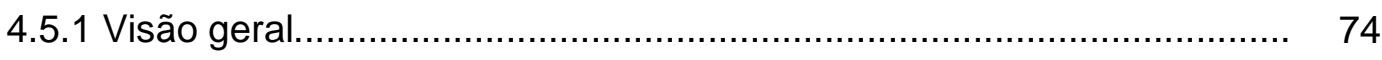

4.5.2 Visão por categoria................................................................. 78

4.6 Considerações finais sobre o capítulo.................................................. 113

6. Conclusões e recomendações............................................................... 113

Referências bibliográficas..................................................................... 121

Apêndice 1 - Instrumento da pesquisa de campo................................... 130 


\section{Lista de figuras}

Figura 1.1 - Selos de certificação orgânica.................................................... 21

Figura 1.2 - Diagrama de pesquisa, seus componentes e métodos................. 22

Figura 3.1 - Exemplo de rotulagem de alimento embalado no Canadá............ 60

Figura 4.1 - Composição da amostra e da base de dados analisados............. 73

Figura 4.2 - Categoria "Conservas e Antepastos": não conformidades por ausência ou inadequação dos itens de rotulagem....................... 82

Figura 4.3 - Não-conformidades referentes a informações obrigatórias: "prazo de validade" impresso em material inapropriado (rótulo à esquerda), ausência de "lote" e da expressão "contém glúten ou não contém glúten" (rótulo à direita)

Figura 4.4 - Informações obrigatórias: "prazo de validade" e "lote" expressos de maneira correta. Local claro e com indicativo, facilitando a visualização pelo consumidor.

Figura 4.5 - Não conformidades em relação às informações obrigatórias "lista de ingredientes", expressão "contém glúten" ou "não contém glúten", "informação nutricional". Etiquetas anexas ao produto....

Figura 4.6 - Não conformidades em relação à expressão "orgânico". Tamanho de letra superior às denominações dos produtos.

Figura 4.7 - A Andicação "orgânico" expressa nos dois rótulos de forma correta.

Figura 4.8 - Rótulos com informações sobre o modo de conservação: o da esquerda, com a informação conforme. $O$ da esquerda encontrase sem a expressão "modo de conservação".

Figura 4.9 - Rótulo com dois selos de certificação orgânica no painel principal

Figura 4.10 - Categoria "Bebidas": não conformidades por ausência ou inadequação dos itens de rotulagem.

Figura 4.11 - Rótulo sem a expressão obrigatória "ingredientes" (à esquerda) e rótulo correto (à direita)......

Figura 4.12 - Rótulo à esquerda com informação incorreta "cultivo ecológico" e o da direita com a expressão "orgânico" disposta corretamente....

Figura 4.13 - Rótulos com denominação de venda do produto fora da zona central do painel principal (à esquerda) e na posição correta (à direita)....

Figura 4.14 - Rótulo com denominação de lote com baixa visibilidade e clareza (à esquerda) e com a informação correta (à direita).

Figura 4.15 - Rótulos do mesmo produto com denominação de lote com baixa visibilidade e clareza (à esquerda) e com a informação correta (à direita).....

Figura 4.16 - Rótulo de produto orgânico importado com rotulagem de acordo com a regulamentação brasileira.

Figura 4.17 - Categoria "Confeitaria e chocolataria": não conformidades por ausência ou inadequação dos itens de rotulagem. 
Figura 4.18 - Informações obrigatórias "lote" e "prazo de validade" nos rótulos de produtos da categoria "Confeitaria e chocolataria".

Figura 4.19 - Rótulo com ausência das informações obrigatórias "identificação de origem" e "lote" (à esquerda) e rótulo com informações corretas (à direita)

Figura 4.20 - Rótulo sem o selo oficial de certificação (à esquerda) e outro com o selo (à direita).

Figura 4.21 - Informações obrigatórias "lote" e "prazo de validade" nos rótulos de produtos da categoria "Confeitaria e chocolataria": inadequações versus informações corretas.

Figura 4.22 - Categoria "Chás": não conformidades por ausência ou inadequação dos itens de rotulagem.

Figura 4.23 - Rótulos da categoria "chás": um sem selo e outro com selo da certificadora.

Figura 4.24 - Informações obrigatórias "lote" e "prazo de validade" nos rótulos de produtos da categoria "Chás": inadequações versus informações corretas

Figura 4.25 - Expressão "orgânico" nos rótulos de produtos da categoria "Chás": inadequação versus informação correta

Figura 4.26 - Categoria "Laticínios": não conformidades por ausência ou inadequação dos itens de rotulagem.

Figura 4.27 - Rótulos de produtos da categoria "Laticínios": inadequação quanto à lista de ingredientes (à esquerda) versus informação correta (à direita).

Figura 4.28 - Mais exemplos de rótulos de produtos da categoria "Laticínios": inadequação quanto à lista de ingredientes (à esquerda) versus informação correta (à direita)

Figura 4.29 - Rótulos de produtos da categoria "Laticínios" com ausência e presença da expressão "orgânico".

Figura 4.30 - Categoria "Barras": não conformidades por ausência ou inadequação dos itens de rotulagem.

Figura 4.31 - Rótulos de produtos da categoria "Barras": inadequação quanto à informação "prazo de validade" (à esquerda) versus informação correta (à direita).

Figura 4.32 - Rótulos de produtos da categoria "Barras": inadequação quanto à informação "prazo de validade" (à esquerda) versus informação correta (à direita).

Figura 4.33 - Categoria "Biscoitos e snacks": não conformidades por ausência ou inadequação dos itens de rotulagem.

Figura 4.34 - Rótulos de produtos da categoria "Biscoitos e snacks": inadequação quanto às informações "lote" e "prazo de validade" (à esquerda) versus informação correta (à direita).....

Figura 4.35 - Rótulos de produtos da categoria "Biscoitos e snacks": inadequação quanto à informação "conteúdo líquido/conteúdo drenado" versus exemplo de informação correta.

Figura 4.36 - Rótulos de produtos da categoria "Biscoitos e snacks": inadequação quanto à informação "prazo de validade" (à esquerda) versus informação correta (à direita). 


\section{Lista de quadros}

Quadro 2.1 - Histórico da regulamentação para alimentos orgânicos no Brasil: 1994 - 2002.

Quadro 2.2 - Histórico da regulamentação para alimentos orgânicos no Brasil:

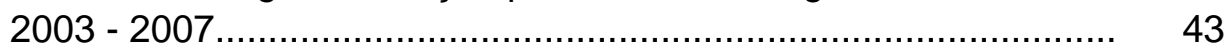

Quadro 2.3 - Certificação por auditoria........................................................ 47

Quadro 2.4 - Sistema participativo de garantia............................................. 48

Quadro 2.5 - Distribuição estadual dos organismos certificadores (OCs).......... 49

Quadro 3.1 - Informações obrigatórias dos países selecionados....................... 66 


\section{Lista de tabelas}

Tabela 4.1 - Composição da amostra e da base de dados analisados............ 72

Tabela 4.2 - Não-conformidades pela ausência da informação nos rótulos de produtos orgânicos analisados: visão geral......

Tabela 4.3 - Inadequações das informações nos rótulos de produtos orgânicos analisados: visão geral.

Tabela 4.4 - Síntese da avaliação dos rótulos dos alimentos orgânicos embalados.

Tabela 4.5 -- Ausência e inadequações das informações nos rótulos de alimentos da categoria "Conservas e antepastos"

Tabela 4.6 -- $\quad$ Ausência e inadequações das informações nos rótulos de alimentos da categoria "Bebidas".

Tabela 4.7 - Ausência de informações e inadequações das informações nos rótulos de alimentos da categoria "Confeitaria e chocolataria"

Tabela 4.8 - $\quad$ Ausência de informações e inadequações das informações nos rótulos de alimentos da categoria "Chás".

Tabela 4.9 - $\quad$ Ausência de informações e inadequações das informações nos rótulos de alimentos da categoria "Laticínios".

Tabela 4.10 - Ausência de informações e inadequações das informações nos rótulos de alimentos da categoria "Barras".

Tabela 4.11 - Ausência de informações e inadequações das informações nos rótulos de alimentos da categoria "Biscoitos e snacks". 


\section{Lista de siglas e acrônimos}

$\begin{array}{ll}\text { ABIA } & \text { Associação Brasileira da Indústria e Alimentação } \\ \text { ABNT } & \text { Associação Brasileira de Normas Técnicas } \\ \text { ACCC } & \text { Comissão Australiana de Competição e do Consumidor } \\ \text { ANMI } & \text { Australian National Measurement Institute } \\ \text { ANVISA } & \text { Agência Nacional de Vigilância Sanitária } \\ \text { APCC } & \text { Plano de Análise de Perigos e Pontos Críticos de Controle } \\ \text { APPCC } & \text { Análise de Perigos e Pontos Críticos de Controle } \\ \text { BP } & \text { Boas Práticas } \\ \text { BPF } & \text { Boas Práticas da Fabricação } \\ \text { BRASILBIO } & \text { Associação Brasileira de Orgânicos } \\ \text { CCAB } & \text { Comitê do Codex Alimentarius do Brasil } \\ \text { CDC } & \text { Código de Defesa do Consumidor } \\ \text { CIAA } & \text { Confederação Europeia da Indústria do Alimento e da Bebida } \\ \text { CNA } & \text { Confederação Nacional da Agricultura } \\ \text { CNI } & \text { Confederação Nacional da Indústria } \\ \text { CNPOrg } & \text { Comissão Nacional da Produção Orgânica } \\ \text { CONSEA } & \text { Conselho Nacional de Segurança Alimentar e Nutricional } \\ \text { CPOrg-UF } & \text { Comissões da Produção Orgânica nas Unidades da } \\ & \text { Federação } \\ \text { CPOrg-UF } & \text { Comissões da Produção Orgânica nas Unidades da } \\ \text { FSAO } & \text { Federação } \\ \text { FAO } & \text { Câmara Setorial da Cadeia Produtiva da Agricultura Orgânica } \\ \text { FMEA } & \text { Food and Agricultural Organization } \\ \text { FSANZ } & \text { Failure, Mode, Effect Analysis } \\ \text { GFSI } & \text { Food Stantards Australia New Zealand } \\ \text { GMP } & \text { Global Food Safety Initiative } \\ \text { HACCP } & \text { Good Manufacturing Practices } \\ \text { IBASE } & \text { Hazard Analysis and Critical Control Pointo do Desenvolvimento, Indústria e Comércio } \\ \text { IBGE } & \text { Instituto Brasileiro de Análises Sociais e Econômicas } \\ \text { IDEC } & \text { Instituto Brasileiro de Geografia e Estatística } \\ \text { INMETRO } & \text { Instituto de Defesa do Consumidor } \\ \text { Instituto Nacional de Metrologia, Normalização e Qualidade } \\ \text { Industial }\end{array}$


MF

MJ

MRE

MS

OAC

OC

OCS

OMS

PPR

SEPES

SIF

SISOrg

SPG

SSOP

USA

USDA

Ministério da Fazenda

Ministério da Justiça

Ministério das Relações Exteriores

Ministério da Saúde

Organismos de Avaliação da Conformidade

Organismo Certificador

Organização de Controle Social

Organização Mundial de Saúde

Programa de Pré-Requisitos

Secretaria de Pesca

Serviço de Inspeção Federal

Sistema Brasileiro de Avaliação da Conformidade Orgânica

Sistemas Participativos de Garantia

Sanitation Standard Operating Procedures

United Stated of America

United States Department of Agriculture 